\title{
LINEAR PERTURBATION THEORY FOR STRUCTURED MATRIX PENCILS ARISING IN CONTROL THEORY*
}

\author{
SHREEMAYEE BORA ${ }^{\dagger}$ AND VOLKER MEHRMANN ${ }^{\ddagger}$
}

\begin{abstract}
We investigate the effect of linear perturbations on several structured matrix pencils arising in control theory. These include skew-symmetric/symmetric pencils arising in the computation of optimal $H_{\infty}$ control and linear quadratic control for continuous and discrete time systems.
\end{abstract}

Keywords. $H_{\infty}$ control, linear-quadratic control, Hamiltonian matrix, continuoustime control, discrete-time control, skew-Hamiltonian/Hamiltonian pencil, skew-Hermitian/Hermitian pencil

AMS subject classification. 93B36, 93B40, 49N35, 65F15, 93B52, 93C05.

1. Introduction. In this paper we study the effects of linear perturbations on the spectra of structured matrix pencils arising in control theory. The results that we present complement and generalize general perturbation results for Hamiltonian matrices as they were recently studied in [14] and we also extend results in [21, 22, 23].

Our main motivation arises from the following classical problems in optimal and robust control. Consider a linear constant coefficient dynamical system of the form

$$
E \dot{x}=A x+B u, \quad x\left(\tau_{0}\right)=x^{0},
$$

where $x(\tau) \in \mathbb{C}^{n}$ is the state, $x^{0}$ is an initial vector, $u(\tau) \in \mathbb{C}^{m}$ is the control input of the system and the matrices $E, A \in \mathbb{C}^{n, n}, B \in \mathbb{C}^{n, m}$ are constant. Here we discuss only the case that the matrix $E$ is nonsingular, thus we allow implicit systems but we do not discuss descriptor systems.

The objective in linear quadratic optimal control, see e.g. $[12,17]$ is to find a control law $u(\tau)$ such that the closed loop system is asymptotically stable and such that the performance criterion

$$
\mathcal{S}(x, u)=\int_{\tau_{0}}^{\infty}\left[\begin{array}{l}
x(\tau) \\
u(\tau)
\end{array}\right]^{T}\left[\begin{array}{cc}
Q & S \\
S^{H} & R
\end{array}\right]\left[\begin{array}{l}
x(\tau) \\
u(\tau)
\end{array}\right] d \tau
$$

is minimized, where $Q=Q^{H} \in \mathbb{C}^{n, n}, R=R^{H} \in \mathbb{C}^{m, m}$ is positive definite and $\left[\begin{array}{cc}Q & S \\ S^{H} & R\end{array}\right]$ is positive semidefinite. Here $A^{H}$ denotes the transpose of the complex conjugate of $A \in \mathbb{C}^{n, n}$.

Application of the maximum principle $[17,20]$ leads to the problem of finding a stable solution to the two-point boundary value problem of Euler-Lagrange equations

$$
N_{c}\left[\begin{array}{c}
\dot{\mu} \\
\dot{x} \\
\dot{u}
\end{array}\right]=H_{c}\left[\begin{array}{l}
\mu \\
x \\
u
\end{array}\right], \quad x\left(\tau_{0}\right)=x^{0}, \quad \lim _{\tau \rightarrow \infty} \mu(\tau)=0,
$$

* This work was completed during the stay of the first author at the Institut für Mathematik, TU Berlin. The authors were partially supported by Deutsche Forschungsgemeinschaft, Research Grant Me 790/11-3.

${ }^{\dagger}$ Mathematical Sciences Division, Institute of Advanced Study in Science and Technology (IASST), Guwahati 781035, Assam, India; bora@math.tu-berlin.de, shreemayee@yahoo.com.

${ }^{\ddagger}$ Institut für Mathematik, Ma 4-5, TU Berlin, Straße des 17. Juni 136, D-10623 Berlin, FRG; mehrmann@math.tu-berlin.de. 
with the matrix pencil

$$
H_{c}-\lambda N_{c}:=\left[\begin{array}{ccc}
0 & A & B \\
A^{H} & Q & S \\
B^{H} & S^{H} & R
\end{array}\right]-\lambda\left[\begin{array}{ccc}
0 & E & 0 \\
-E^{H} & 0 & 0 \\
0 & 0 & 0
\end{array}\right] .
$$

It is well known that the finite eigenvalues of $H_{c}-\lambda N_{c}$ are symmetric with respect to the imaginary axis (and if the problem is real then also with respect to the real axis). If $E$ is invertible, then under the usual control theoretic assumptions [17, 26, 27], this pencil has exactly $n$ eigenvalues in the left half plane and $n$ eigenvalues in the right half plane plus $m$ infinite eigenvalues. Clearly then the pencil has a unique deflating subspace associated with the eigenvalues in the open left half complex plane. If $E$ or $R$ are not invertible, then the situation is more complex and different approaches can be taken, $[4,6,7,17]$. In this paper we discuss mainly the case that $E$ and $R$ are invertible.

The solution of the boundary value problem (1.3) can be obtained in many different ways. The approach in most computer aided control design packages is to decouple the boundary value problem via the computation of the solution of an associated algebraic Riccati equation. But one may also directly solve the boundary value problem (1.3) by computing the generalized Schur-form of the pencil $H_{c}-\lambda N_{c}$, $[2,17,27,26]$, i.e., one determines unitary matrices $P, Q \in \mathbb{C}^{2 n+m, 2 n+m}$, such that

$$
P N_{c} Q=\left[\begin{array}{ccc}
N_{11} & N_{12} & N_{13} \\
0 & N_{22} & N_{23} \\
0 & 0 & N_{33}
\end{array}\right], P H_{c} Q=\left[\begin{array}{ccc}
H_{11} & H_{12} & H_{13} \\
0 & H_{22} & H_{23} \\
0 & 0 & H_{33}
\end{array}\right],
$$

where the subpencil $H_{11}-\lambda N_{11}$ has all its eigenvalues in the left half plane, to decouple the forward and backward integration in the boundary value problem.

In this paper we study the perturbation theory for the eigenvalue problem (1.4). For this several different types of perturbations should be considered as separate cases. If one uses classical methods that do not preserve the structure, like the $Q Z$ algorithm [9], to compute the generalized Schur form in finite precision arithmetic, then the special structure of the pencil is ignored and hence the whole matrices $H_{c}, N_{c}$ are subject to perturbations. We do not discuss this case here, since it is well analyzed in the monograph [24].

If one studies perturbation theory in order to deal with uncertainties in the data of the system, then the blocks $E, A, B, Q, S, R$ are subject to perturbations of only the blocks $E, A, B$, since typically the matrices of the cost function are free to be chosen under the constraints that $\left[\begin{array}{cc}Q & S \\ S^{H} & R\end{array}\right]$ is positive semidefinite and $R$ positive definite. Also one may study the particular case that $E=I$ is not perturbed.

In all cases it is essential to analyze whether the perturbations can lead to eigenvalues on the imaginary axis, in which case the spectral symmetry and the uniqueness of the deflating subspace associated with the open left half plane may be lost, see $[8,17,21,22,23]$.

It is well-known, see $[17,18]$, that the discrete-time analogue to the linear quadratic control problem leads to slightly different matrix pencils of the form

$$
H_{d}-\lambda N_{d}=\left[\begin{array}{ccc}
0 & A & B \\
-E^{H} & Q & S \\
0 & S^{H} & R
\end{array}\right]-\lambda\left[\begin{array}{ccc}
0 & E & 0 \\
-A^{H} & 0 & 0 \\
-B^{H} & 0 & 0
\end{array}\right] .
$$


Here the spectral symmetry is with respect to the unit circle, i.e. the finite eigenvalues come in pairs $\lambda, \frac{1}{\lambda}$ or quadruples $\lambda, \bar{\lambda}, \frac{1}{\lambda}, \frac{1}{\lambda}$ in the case of real matrices. The perturbation problems can be discussed analogously and here the important question that arises is the study of perturbations which lead to eigenvalues on the unit circle, where again the spectral symmetry and the uniqueness of the deflating subspace associated with the eigenvalues in the open unit disk may be disturbed.

The second motivation comes from the optimal $H_{\infty}$-control problem which arises in the context of robust control in frequency domain, see, e.g., the recent monographs $[10,28]$. In the context of the so called $\gamma$-iteration, in the newly developed approach suggested in [5], generalized Schur forms have to be computed for matrix pencils of the form

$$
\hat{H}_{c}(t)-\lambda \hat{N}_{c}:=\left[\begin{array}{ccc}
0 & A & B \\
A^{H} & 0 & S \\
B^{H} & S^{H} & R(t)
\end{array}\right]-\lambda\left[\begin{array}{ccc}
0 & E & 0 \\
-E^{H} & 0 & 0 \\
0 & 0 & 0
\end{array}\right]
$$

with an indefinite Hermitian matrix

$$
R(t)=\left[\begin{array}{cc}
R_{11}-t I & R_{12} \\
R_{12}^{H} & R_{22}
\end{array}\right]
$$

which varies with the positive parameter $t$ (playing the role of the parameter $\gamma$ in the $\gamma$-iteration), while the other coefficients are constant in $t$. Here besides the classical questions of perturbation theory as above, we are interested in the eigenvalues and deflating subspaces as functions of $t$ and we want to study the size of perturbations that is needed to bring any of the finite eigenvalues to the imaginary axis.

Again there is a discrete-time $H_{\infty}$ analogue to this case [10] which leads to matrix pencils

$$
\hat{H}_{d}(t)-\lambda \hat{N}_{d}=\left[\begin{array}{ccc}
0 & A & B \\
-E^{H} & 0 & S \\
0 & S^{H} & R(t)
\end{array}\right]-\lambda\left[\begin{array}{ccc}
0 & E & 0 \\
-A^{H} & 0 & 0 \\
-B^{H} & 0 & 0
\end{array}\right]
$$

Here again we are interested in the eigenvalues and deflating subspaces as functions of $t$ and we want to study the size of perturbations that is needed to bring any of the finite eigenvalues to the unit circle.

The paper is organized as follows. First we introduce the notation and give some preliminary results in Section 2. In Section 3 we formulate a framework for analyzing the effect of linear perturbations on general matrix pencils. We then study the special cases of perturbations for general skew-symmetric/symmetric pencils arising from continuous-time problems in Section 4 and the corresponding discrete-time problems in Section 5.

2. Notation and preliminaries. We denote the set of all complex (real) matrices of size $n$ by $\mathbb{C}^{n, n}\left(\mathbb{R}^{n, n}\right)$. Given a matrix $A$, we denote its complex conjugate by $\bar{A}$, its transpose by $A^{T}$ and the transpose of its complex conjugate by $A^{H}$. We denote the identity matrix of size $n$ by $I_{n}$. Also we consider the 'flip' permutation matrix

$$
F_{n}:=\left[\begin{array}{ccccc}
0 & 0 & \cdots & 0 & 1 \\
0 & 0 & \cdots & 1 & 0 \\
\vdots & \vdots & \ddots & \vdots & \vdots \\
0 & 1 & \cdots & 0 & 0 \\
1 & 0 & \cdots & 0 & 0
\end{array}\right] \in \mathbb{C}^{n, n}
$$


We denote the spectrum of a square matrix $A$ and a pencil $(A, B)$ by $\sigma(A)$ and $\sigma(A, B)$, respectively. Given a set $S$ we denote its boundary by $\partial S$. For $A \in \mathbb{C}^{n, n}$, we define the spectral radius of $A$ as $r(A):=\max \{|\lambda|: \lambda \in \sigma(A)\}$.

Also, given $z \in \mathbb{C}$, we define

$$
\operatorname{sep}_{\mathbb{R}}(z, \Delta H, \Delta N):=\min \{|t|: z \in \sigma(H+t \Delta H, N+t \Delta N), t \in \mathbb{R}\} .
$$

It is well known (see e.g.[25]) that for every matrix $A \in \mathbb{C}^{n, n}\left(\mathbb{R}^{n, n}\right)$ there exist symmetric matrices $T=T^{T}$ and $S=S^{T}$, where $S$ is also nonsingular, such that $A=T S^{-1}$. Note that if $A$ is not real then these factors in general are complex symmetric but not Hermitian. Furthermore, if $A \in \mathbb{R}^{n, n}$, then $T$ and $S$ can be chosen to be real matrices. Since this result is due to Frobenius, we refer to $T$ and $S$ as Frobenius factors of $A$. In our work, we will need similar factorizations, however with Frobenius factors that are Hermitian. It is easy to see that if $A \in \mathbb{R}^{n, n}$, then $A$ always has Hermitian Frobenius factors. However, if $A \in \mathbb{C}^{n, n}$, then Hermitian factors need not exist. This follows by observing the fact that if $A=T S^{-1}$ with $T^{H}=T$ and $S^{H}=S$, then we must have $A S=S A^{H}$, that is $A=S A^{H} S^{-1}$. This, implies that the matrices $A$ and $A^{H}$ must be similar and hence they must have the same eigenvalues. Thus, a necessary condition for the existence of Hermitian Frobenius factors $T$ and $S$ is that $\sigma(A)=\sigma\left(A^{H}\right)$.

We show that $\sigma(A)=\sigma\left(A^{H}\right)$ is also a sufficient condition for $A$ to have Hermitian Frobenius factors. For this, we first observe that $\sigma(A)=\sigma\left(A^{H}\right)$ implies that for every non-real eigenvalue of $A$ its complex conjugate is also an eigenvalue with the same multiplicity.

Proposition 2.1. Let $A \in \mathbb{C}^{n, n}$ be such that $\sigma(A)=\sigma\left(A^{H}\right)$. Let $\eta_{1}, \eta_{2}, \ldots, \eta_{r}$ be the pairwise distinct real eigenvalues of $A$ and let $\lambda_{1}, \lambda_{2}, \ldots, \lambda_{p}, \overline{\lambda_{1}}, \overline{\lambda_{2}}, \ldots, \overline{\lambda_{p}}$, be the pairwise distinct non-real eigenvalues of $A$. Furthermore, let $m_{1}, m_{2}, \ldots, m_{p}$ be the multiplicities of the eigenvalues $\lambda_{1}, \lambda_{2}, \ldots, \lambda_{p}$, respectively such that $\sum_{i=1}^{p} m_{i}=m$, and let $k_{1}, k_{2}, \ldots, k_{r}$ be the multiplicities of $\eta_{1}, \eta_{2}, \ldots, \eta_{r}$, such that $\sum_{j=1}^{r} k_{j}=k$, and $n=2 m+k$. Then, with the permutation matrix

$$
U=\operatorname{diag}\left(F_{2 m_{1}}, F_{2 m_{2}}, \ldots, F_{2 m_{p}}, F_{n_{1}}, F_{n_{2}}, \ldots, F_{n_{s}}\right),
$$

we have that $A$ and $A^{H}$ have the Jordan decompositions

$$
A=P J P^{-1}, A^{H}=\left(P^{-H} U\right) \bar{J}\left(P^{-H} U\right)^{-1},
$$

where the blocks are ordered as in

$$
\begin{gathered}
J:=\operatorname{diag}\left(J_{m_{1}}\left(\lambda_{1}\right), J_{m_{1}}\left(\overline{\lambda_{1}}\right), \ldots, J_{m_{p}}\left(\lambda_{p}\right), J_{m_{p}}\left(\overline{\lambda_{p}}\right),\right. \\
\left.J_{n_{1}}\left(\eta_{1}\right), J_{n_{2}}\left(\eta_{2}\right), \ldots, J_{n_{r}}\left(\eta_{r}\right)\right),
\end{gathered}
$$

such that for $i=1,2, \ldots, p, j=1,2, \ldots, r$,

$$
J_{m_{i}}\left(\lambda_{i}\right):=\left[\begin{array}{cccccc}
\lambda_{i} & \varphi & 0 & \cdots & 0 & 0 \\
0 & \lambda_{i} & \varphi & \cdots & 0 & 0 \\
\vdots & \vdots & \vdots & \ddots & \vdots & \vdots \\
0 & 0 & 0 & \cdots & \lambda_{i} & \varphi \\
0 & 0 & 0 & \cdots & 0 & \lambda_{i}
\end{array}\right]
$$


and

$$
J_{n_{j}}\left(\eta_{j}\right):=\left[\begin{array}{cccccc}
\eta_{j} & \varphi & 0 & \cdots & 0 & 0 \\
0 & \eta_{j} & \varphi & \cdots & 0 & 0 \\
\vdots & \vdots & \vdots & \ddots & \vdots & \vdots \\
0 & 0 & 0 & \cdots & \eta_{j} & \varphi \\
0 & 0 & 0 & \cdots & 0 & \eta_{j}
\end{array}\right]
$$

with $\varphi=0$ or $\varphi=1$ and $U$ satisfies $\bar{J} U-U J^{H}=0$.

Proof. Let $A=P J P^{-1}$ with $J$ as in (2.1) be a Jordan decomposition of $A$. Then, $A^{H}=P^{-H} J^{H} P^{H}$ with

$$
\begin{gathered}
J^{H}=(\bar{J})^{T}=\operatorname{diag}\left(J_{m_{1}}\left(\overline{\lambda_{1}}\right), J_{m_{1}}\left(\lambda_{1}\right), \ldots, J_{m_{p}}\left(\overline{\lambda_{p}}\right), J_{m_{p}}\left(\lambda_{p}\right),\right. \\
\left.J_{n_{1}}\left(\eta_{1}\right), J_{n_{2}}\left(\eta_{2}\right), \ldots, J_{n_{r}}\left(\eta_{r}\right)\right)^{T},
\end{gathered}
$$

and for $i=1,2, \ldots, p$,

$$
\begin{aligned}
& {\left[\begin{array}{cc}
J_{m_{i}}\left(\overline{\lambda_{i}}\right) & 0 \\
0 & J_{m_{i}}\left(\lambda_{i}\right)
\end{array}\right] F_{2 m_{i}}=\left[\begin{array}{ccccc}
\overline{\lambda_{i}} & \frac{\varphi}{\lambda_{i}} & \varphi & & \\
& & \ddots & \ddots & \\
& & & \lambda_{i} & \varphi \\
& & & & \lambda_{i}
\end{array}\right]\left[\begin{array}{ccccc}
0 & 0 & \cdots & 0 & 1 \\
0 & 0 & \cdots & 1 & 0 \\
\vdots & \vdots & \ddots & \vdots & \vdots \\
0 & 1 & \cdots & 0 & 0 \\
1 & 0 & \cdots & 0 & 0
\end{array}\right]} \\
& =\left[\begin{array}{cccccc}
0 & 0 & \cdots & 0 & \varphi & \overline{\lambda_{i}} \\
0 & 0 & \cdots & \varphi & \overline{\lambda_{i}} & 0 \\
\vdots & \vdots & \ddots & \vdots & \vdots & \vdots \\
\varphi & \lambda_{i} & \cdots & 0 & 0 & 0 \\
\overline{\lambda_{i}} & 0 & \cdots & 0 & 0 & 0
\end{array}\right] \\
& =\left[\begin{array}{ccccc}
0 & 0 & \cdots & 0 & 1 \\
0 & 0 & \cdots & 1 & 0 \\
\vdots & \vdots & \ddots & \vdots & \vdots \\
0 & 1 & \cdots & 0 & 0 \\
1 & 0 & \cdots & 0 & 0
\end{array}\right]\left[\begin{array}{ccccc}
\overline{\lambda_{i}} & & & & \\
\varphi & \overline{\lambda_{i}} & & & \\
& \ddots & \ddots & & \\
& & \varphi & \lambda_{i} & \\
& & \varphi & \lambda_{i}
\end{array}\right] \\
& =F_{2 m_{i}}\left[\begin{array}{cc}
J_{m_{i}}\left(\overline{\lambda_{i}}\right) & 0 \\
0 & J_{m_{i}}\left(\lambda_{i}\right)
\end{array}\right]^{T}
\end{aligned}
$$

Similarly for $j=1,2, \ldots, s$, we have $J_{n_{j}} F_{n_{j}}=F_{n_{j}} J_{n_{j}}^{T}$ and thus it follows that $\bar{J} U$ $U J^{H}=0$.

Using Proposition 2.1 together with Theorem 12.5.1 of [16] we then construct a nonsingular Hermitian solution $S$ of the equation $A S-S A^{H}=0$.

Theorem 2.2. Let $A \in \mathbb{C}^{n, n}$ be such that $\sigma(A)=\sigma\left(A^{H}\right)$. Then there exists a nonsingular Hermitian matrix $S$ such that $A S=S A^{H}$.

Proof. Using the notation of Proposition 2.1, it follows by Theorem 12.5.1 in [16] that all solutions of the equation $A S-S A^{H}=0$ are of the form $S=$ $P Y\left(P^{-H} U\right)^{-1}=P Y U P^{H}$. Here $Y$ satisfies $J Y-Y \bar{J}=0$ and has the block-form $Y=\operatorname{diag}\left(Y_{1}, Y_{2}, \ldots, Y_{p}, I_{k}\right)$, where $Y_{i}=\left[\begin{array}{cc}0 & Y_{i, i} \\ Y_{i, i} & 0\end{array}\right] \in \mathbb{C}^{2 m_{i}, 2 m_{i}}$, each $Y_{i, i}$ being an 
arbitrary upper triangular Toeplitz matrix for $i=1,2, \ldots, p$. Since we want $Y U$ to be Hermitian, a possible choice is $Y_{i, i}=I_{m_{i}}$. Then for $i=1,2, \ldots, p$, we have

$$
Y_{i} F_{2 m_{i}}=\operatorname{diag}\left(F_{m_{i}}, F_{m_{i}}\right)=F_{2 m_{i}} Y_{i},
$$

which implies that $(Y U)^{H}=U^{H} Y^{H}=U Y=Y U$. Thus, $S:=P Y U P^{H}$ is nonsingular and Hermitian.

Theorem 2.2 immediately provides a necessary and sufficient condition for $A \in$ $\mathbb{C}^{n, n}$ to have Hermitian Frobenius factors.

Corollary 2.3. Given $A \in \mathbb{C}^{n, n}$, there exist Hermitian matrices, $T$ and $S$ where $S$ is also nonsingular such that $A=T S^{-1}$ if and only if $\sigma(A)=\sigma\left(A^{H}\right)$.

Proof. Suppose that there exist Hermitian matrices $T$ and $S$, where $S$ is also nonsingular such that $A=T S^{-1}$. Then $T=A S$ and $T=T^{H}$ and hence $A S=S A^{H}$ or $A=S A^{H} S^{-1}$, i.e., $\sigma(A)=\sigma\left(A^{H}\right)$. For the converse, suppose that $\sigma(A)=\sigma\left(A^{H}\right)$. Then by Theorem 2.2, there exists a nonsingular Hermitian matrix $S$ such that $A S=$ $S A^{H}$. This implies that $A=\left(S A^{H}\right) S^{-1}$ and the proof follows by setting $T=S A^{H}$.

Since the factorization $A=T S^{-1}$ with $S^{H}=S$ and $T^{H}=T$, if it exists, depends on the choice of $S$ as a solution of $A X-X A^{H}=0$, it is evident from Theorem 2.2 that this factorization is, in general, not unique.

In the following we will need Frobenius factorizations for matrices that depend on a complex parameter. Suppose that $A$ depends smoothly upon a complex parameter $z$ and $\sigma(A(z))=\sigma\left(A(z)^{H}\right)$, and let $A(z)=T(z) S(z)^{-1}$ be a Frobenius factorization of $A(z)$ with $T(z)=T(z)^{H}$ and $S(z)=S(z)^{H}$. Using the spectral factorization of $T(z)$, there exists a unitary matrix $U(z)$ such that

$$
T(z)=U(z)\left[\begin{array}{ccc}
D_{+}(z) & & \\
& D_{-}(z) & \\
& & 0
\end{array}\right] U(z)^{H},
$$

where $D_{+}(z) \in \mathbb{C}^{\pi, \pi},-D_{-}(z) \in \mathbb{C}^{\nu, \nu}$ are diagonal matrices with positive diagonal elements and $\pi(z) \geq \nu(z)$ where $(\pi(z), \nu(z), \omega(z))$ with $\pi(z)+\nu(z)+\omega(z)=n$ is the inertia-index of $T(z)$, see [16].

Setting

$Q(z):=U(z)\left[\begin{array}{lll}\left(D_{+}(z)\right)^{\frac{1}{2}} & & \\ & \left(-D_{-}(z)\right)^{\frac{1}{2}} & \\ & I_{\omega}(z)\end{array}\right], \tilde{I}_{T}(z):=\left[\begin{array}{lll}I_{\pi}(z) & & \\ & -I_{\nu}(z) & \\ & & 0\end{array}\right]$,

we have for given $z$ a factorization

$$
A(z)=Q(z) \tilde{I}_{T}(z) Q^{H}(z) S(z)^{-1} .
$$

Note that the choice $\pi \geq \nu$ makes the matrix $\tilde{I}_{T}(z)$ unique, while there is still much freedom in the choice of the transformation matrix $Q(z)$. In an analogous way we can construct a factorization

$$
A(z)=T(z)\left(V(z)\left(\tilde{I}_{S}(z)\right) V(z)^{H}\right)^{-1}=T(z) V(z)^{-H}\left(\tilde{I}_{S}(z)\right) V(z)^{-1}
$$

by using the spectral factorization and the inertia index of $S(z)$.

An interesting open question that one may discuss in this context is how to obtain a smooth Frobenius factorization, when the matrix depends smoothly on a parameter as in our case. 
3. Linear perturbation of general matrix pencils. In this section we consider the effect of perturbing a regular square matrix pencil $(H, N)$ where $H, N \in$ $\mathbb{C}^{n, n}\left(\mathbb{R}^{n, n}\right)$ by linear perturbations $(H+t \Delta H, N+t \Delta N)$. Here $\Delta H, \Delta N \in \mathbb{C}^{n, n}\left(\mathbb{R}^{n, n}\right)$ are fixed perturbation matrices and the parameter $t$ varies over the real numbers.

Lemma 3.1. For every $z \in \mathbb{C}$ we have $\operatorname{sep}_{\mathbb{R}}(z, \Delta H, \Delta N)<\infty$ if and only if $(\Delta H-$ $z \Delta N)(H-z N)^{-1}$ has a non-zero real eigenvalue. Moreover, if $\operatorname{sep}_{\mathbb{R}}(z, \Delta H, \Delta N)<\infty$, then

$$
\operatorname{sep}_{\mathbb{R}}(z, \Delta H, \Delta N)=\left[\max _{\lambda \in \mathbb{R}}\left\{\lambda \in \sigma\left((\Delta H-z \Delta N)(H-z N)^{-1}\right)\right\}\right]^{-1} .
$$

Proof. The proof follows immediately from the fact that for $\lambda \notin \sigma(H, N)$, we have

$$
H+t \Delta H-\lambda(N+t \Delta N)=\left[I+t(\Delta H-\lambda \Delta N)(H-\lambda N)^{-1}\right](H-\lambda N) .
$$

Hence $\lambda \in \sigma(H+t \Delta H, N+t \Delta N)$ if and only if $-1 / t \in \sigma\left((\Delta H-\lambda \Delta N)(H-\lambda N)^{-1}\right)$.

As discussed in the introduction, we are interested in conditions which guarantee that all the eigenvalues of the perturbed pencils $(H+t \Delta H, N+t \Delta N), t \in \mathbb{R}$, remain within a particular open subset, say $\mathbb{C}_{g}$, of the complex plane. Since the eigenvalues of $(H+t \Delta H, N+t \Delta N)$ move continuously as $t$ varies in $\mathbb{R}$, the smallest value of $|t|$ for which these eigenvalues move out of $\mathbb{C}_{g}$ is evidently equal to

$$
r\left(\mathbb{C}_{g}, \Delta H, \Delta N\right):=\inf _{z \in \delta \mathbb{C}_{g}} \operatorname{sep}(z, \Delta H, \Delta N) .
$$

Note that similar distances are very important in other contexts of control theory, where the smallest perturbation that makes a system unstable is called the stability radius [11] and the smallest perturbation that makes a system non-passive is called the passivity radius [19].

Since a complex number $z$ becomes an eigenvalue of the perturbed pencil $(H+$ $t \Delta H, N+t \Delta N)$ for some $t \in \mathbb{R}$ if and only if the matrix $(\Delta H-z \Delta N)(H-z N)^{-1}$ has a non-zero real eigenvalue, it is possible that there exist pencils $(H, N)$ with corresponding perturbations $(\Delta H, \Delta N)$ and sets $\mathbb{C}_{g}$ such that $r\left(\mathbb{C}_{g}, \Delta H, \Delta N\right)=\infty$, that is, the eigenvalues of the perturbed pencils $(H+t \Delta H, N+t \Delta N)$ always remain inside $\mathbb{C}_{g}$ as $t$ varies over the real numbers. In such cases, $\operatorname{sep}(z, \Delta H, \Delta N)=\infty$ for all $z \in \partial \mathbb{C}_{g}$. This is illustrated by the following example.

Example 3.2. Consider the pencil $(H, N)$ where

$$
H:=\left[\begin{array}{ll}
1 & 2 \\
2 & 1
\end{array}\right] \text {, and } N:=\left[\begin{array}{cc}
0 & 1 \\
-1 & 0
\end{array}\right] .
$$

Its eigenvalues are $\sqrt{3}$ and $-\sqrt{3}$. Let

$$
\Delta H:=\left[\begin{array}{cc}
1 & 0 \\
0 & -1
\end{array}\right], \Delta N:=0
$$

be the perturbations to $H$ and $N$, respectively.

Let $\mathbb{C}_{g}:=\mathbb{C} \backslash\{z \in \mathbb{C}: \operatorname{Re}(z)=0\}$. Then the boundary $\partial \mathbb{C}_{g}$ of $\mathbb{C}_{g}$ is evidently the imaginary axis. Therefore, by Lemma 3.1 for all $t \in \mathbb{R}$, the eigenvalues of the pencils $(H+t \Delta H, N+t \Delta N)$ are always in $\mathbb{C}_{g}$, if and only if the matrix

$$
(\Delta H-z \Delta N)(H-z N)^{-1}=\frac{1}{3-z^{2}}\left[\begin{array}{lc}
-1 & 2-z \\
-(2+z) & 1
\end{array}\right]
$$


has no non-zero real eigenvalue for every $z \in \mathbb{C}$ lying on the imaginary axis. The eigenvalues of $(\Delta H-z \Delta N)(H-z N)^{-1}$ are $i / \sqrt{\left(3-z^{2}\right)}$ and $-i / \sqrt{\left(3-z^{2}\right)}$. Now for every $z$ lying on the imaginary axis, there exists a real number $\gamma$, such that $z=i \gamma$. Therefore, for $z \in \partial \mathbb{C}_{g}$, the eigenvalues of $(\Delta H-z \Delta N)(H-z N)^{-1}$ are $i / \sqrt{\left(3+\gamma^{2}\right)}$ and $-i / \sqrt{\left(3+\gamma^{2}\right)}$. This shows that $(\Delta H-z \Delta N)(H-z N)^{-1}$ has no real eigenvalues for all $z \in \partial \mathbb{C}_{g}$. Hence, $\sigma(H+t \Delta H, N+t \Delta N) \subset \mathbb{C}_{g}$, for all $t \in \mathbb{R}$.

Since $z \in \sigma(H+t \Delta H, N+t \Delta N)$ for some $t \in \mathbb{R}$, if and only if the matrix $F(z):=$ $(\Delta H-z \Delta N)(H-z N)^{-1}$ has a real eigenvalue, we identify conditions under which the latter matrix has a real eigenvalue. Under the assumption that $\sigma(F(z))=\sigma\left(F(z)^{H}\right)$, let $F(z)=T(z)\{S(z)\}^{-1}$ be a Frobenius factorization of $F(z)$ where $T(z)^{H}=T(z)$ and $S(z)^{H}=S(z)$. The following result gives a necessary and sufficient condition for this matrix to have a real eigenvalue.

Lemma 3.3. For a fixed $z \in \mathbb{C}$, let $T(z)$ and $S(z)$ be Frobenius factors of $F(z):=(\Delta H-z \Delta N)(H-z N)^{-1}$, where $T(z)^{H}=T(z), S(z)^{H}=S(z)$ and $S(z)$ is nonsingular. Let $\left(\pi_{T}(z), \nu_{T}(z), \omega_{T}(z)\right)$ with $\pi_{T}(z) \geq \nu_{T}(z)$ be the inertia index of $T(z)$. Furthermore, let $\tilde{I}_{T}(z), Q(z)$ be as in a factorization of the form (2.2) of $F(z)$.

Then $(\Delta H-z \Delta N)(H-z N)^{-1}$ has a non-zero real eigenvalue if and only if the matrix $\tilde{I}_{T}(z) Q(z)\{S(z)\}^{-1} Q(z)^{H}$ has a non-zero real eigenvalue.

Proof. The proof follows, since $F(z)$ and $\tilde{I}_{T}(z) Q(z)\{S(z)\}^{-1} Q(z)^{H}$ are similar. $\square$

It is evident that the roles of the matrices $T(z)$ and $S(z)$ in Lemma 3.3 can be interchanged.

Lemma 3.4. For a fixed $z \in \mathbb{C}$, let the matrix $T(z)$ and $S(z)$ be Frobenius factors of $F(z):=(\Delta H-z \Delta N)(H-z N)^{-1}$ such that $T(z)^{H}=T(z), S(z)^{H}=S(z)$ and $S(z)$ is invertible. Let $\left(\pi_{S}(z), \nu_{S}(z), \omega_{S}(z)\right)$ with $\pi_{S}(z) \geq \nu_{S}(z)$ be the inertia index of $S(z)$. Let, furthermore, $\tilde{I}_{S}(z), V(z)$ be the factors in a factorization of the form (2.3) of $F(z)$. Then $(\Delta H-z \Delta N)(H-z N)^{-1}$ has a non-zero real eigenvalue if and only if the matrix $V(z)^{-1} T(z) V(z)^{-H} \tilde{I}_{S}(z)$ has a non-zero real eigenvalue.

In general, the function $\operatorname{sep}_{\mathbb{R}}(z, \Delta H, \Delta N)$ is discontinuous as a function of $z$, since it depends on the matrix $(\Delta H-z \Delta N)(H-z N)^{-1}$ having a real eigenvalue. However, it is possible that given a set $\mathbb{C}_{g} \subset \mathbb{C}$, the structure of the matrices $H, N, \Delta H$, and $\Delta N$ are such that the matrix $(\Delta H-z \Delta N)(H-z N)^{-1}$ always has one or more real eigenvalues for $z \in \partial \mathbb{C}_{g}$. Let these eigenvalues be $h_{1}(z), \ldots, h_{p}(z)$. Then $(\Delta H-$ $z \Delta N)(H-z N)^{-1}$ is an analytic function of $z \in \mathbb{C} \backslash \sigma(H, N)$, and hence in particular of $z \in \partial \mathbb{C}_{g}$ (Theorem 1.5, pp. 66, [13]) and the eigenvalues $h_{1}(z), \ldots, h_{p}(z)$ are continuous (Corollary 3, pp. 105, [3]). Therefore, for such cases we have for $z \in \partial \mathbb{C}_{g}$,

$$
\operatorname{sep}_{\mathbb{R}}(z, \Delta H, \Delta N)=\left\{\max \left\{\left|h_{k}(z)\right|: k=1, \ldots, p\right\}\right\}^{-1},
$$

which implies that $\operatorname{sep}_{\mathbb{R}}(z, \Delta H, \Delta N)$ is a continuous function of $z$. In the special situation that all the eigenvalues of $(\Delta H-z \Delta N)(H-z N)^{-1}$ are real, we have $\operatorname{sep}_{\mathbb{R}}(z, \Delta H, \Delta N)=\left\{r\left((\Delta H-z \Delta N)(H-z N)^{-1}\right)\right\}^{-1}$ for all $z \in \partial \mathbb{C}_{g}$. In such cases, the distribution of the eigenvalues of $(H+t \Delta H, N+t \Delta N)$ on $\partial \mathbb{C}_{g}$ may be analyzed by plotting the level curves of the spectral radius function $r\left((\Delta H-z \Delta N)(H-z N)^{-1}\right)$ in neighbourhoods of $\partial \mathbb{C}_{g}$. Then the smallest value of $|t|$ for which some $z \in \partial \mathbb{C}_{g}$ is an eigenvalue of $(H+t \Delta H, N+t \Delta N)$ is evidently given by the smallest value of $\epsilon$ for which the level set

$$
L(\epsilon, \Delta H, \Delta N):=\left\{z \in \mathbb{C} \backslash \sigma(H, N): r\left((\Delta H-z \Delta N)(H-z N)^{-1}\right)=\epsilon^{-1}\right\}
$$


intersects $\partial \mathbb{C}_{g}$. In other words, for such problems, the distance to the boundary of $\mathbb{C}_{g}$ is given by

$$
r\left(\mathbb{C}_{g}, \Delta H, \Delta N\right):=\min \left\{\epsilon \in \mathbb{R}: L(\epsilon, \Delta H, \Delta N) \cap \partial \mathbb{C}_{g} \neq 0\right\} .
$$

By Proposition 2.1 of [1] the spectral radius function $r\left((\Delta H-z \Delta N)(H-z N)^{-1}\right)$ is non-constant on open subsets of $\mathbb{C} \backslash \sigma(H, N)$. This together with the fact that it is also continuous on $\mathbb{C} \backslash \sigma(H, N)$ implies that the level sets $L(\epsilon, \Delta H, \Delta N)$ are closed sets which have no interior points. In other words, they are curves on the complex plane. Furthermore, the curve $L(\epsilon, \Delta H, \Delta N)$ intersects $\partial \mathbb{C}_{g}$ at only a finite number of points, since at each such point we must either have $z \in \sigma(H+\epsilon \Delta H, N+\epsilon \Delta N)$ or $z \in \sigma(H-\epsilon \Delta H, N-\epsilon \Delta N)$. This justifies the use of 'minimum' instead of 'infimum' in $(3.1)$.

In the following theorem, we give sufficient conditions for all the eigenvalues of the matrix $(\Delta H-z \Delta N)(H-z N)^{-1}$ to be real.

THEOREM 3.5. All the eigenvalues of the matrix $F(z)=(\Delta H-z \Delta N)(H-$ $z N)^{-1}, z \in \mathbb{C}$, are real if there exists a Frobenius factorization $F(z)=T(z) S(z)^{-1}$, with $T(z)^{H}=T(z)$, and $S(z)^{H}=S(z)$ and the Frobenius factors $T(z)$ and $S(z)$ satisfy any of the following conditions.

(i) $T(z)$ and $S(z)^{-1}$ commute.

(ii) $T(z)$ is positive semidefinite.

(iii) $S(z)$ is positive definite.

Proof. (i) Since $(\Delta H-z \Delta N)(H-z N)^{-1}=T(z) S(z)^{-1}$, where $T(z)$ and $S(z)$ are Hermitian, the matrix $(\Delta H-z \Delta N)(H-z N)^{-1}$ is Hermitian if $T(z) S(z)^{-1}=$ $S(z)^{-1} T(z)$ and therefore all its eigenvalues are real. This proves (i).

(ii) If $T(z)$ and $S(z)$ do not commute but $T(z)$ is positive semidefinite with $\pi(z)$ non-zero eigenvalues, then we obtain the Frobenius factorization $(2.2)$ as $F(z)=$ $Q(z) \tilde{I}_{T}(z) Q(z)^{H} S(z)^{-1}$ with $\tilde{I}_{T}(z)=\left[\begin{array}{cc}I_{\pi} & 0 \\ 0 & 0\end{array}\right]$. If we partition

$$
Q(z)^{H} S(z)^{-1} Q(z)=\left[\begin{array}{ll}
S_{11}(z) & S_{12}(z) \\
S_{21}(z) & S_{22}(z)
\end{array}\right]
$$

conformally with $\tilde{I}_{T}(z)$, then $S_{11}(z)$ is Hermitian and

$$
Q(z)^{H} T(z)\{S(z)\}^{-1} Q(z)=\left[\begin{array}{cc}
S_{11}(z) & S_{12}(z) \\
0 & 0
\end{array}\right]
$$

Therefore, $\sigma\left((\Delta H-z \Delta N)(H-z N)^{-1}\right)=\sigma\left(S_{11}(z)\right) \cup\{0\}$ which is real.

(iii) The proof follows as in (ii) by exchanging the roles of $S$ and $T$ and using the factorization (2.3).

Note that Theorem 3.5 also holds if the condition of positive semidefiniteness in (ii) and positive definiteness in (iii) are replaced by negative semidefiniteness and negative definiteness, respectively.

4. Linear perturbation of structured matrix pencils arising from continuous-time control problems. In this section we apply the results from Section 3 to the specific pencils from control theory that we introduced in Section 1. The matrices $H$ and $N$ then have special structure and in order not to destroy the properties of the pencils it should be guaranteed that the perturbations preserve this structure.

This means that we study the effect of perturbations $(H+t \Delta H, N+t \Delta N), t \in \mathbb{R}$, where the matrices $\Delta H$ and $\Delta N$ have the same structure as $H$ and $N$, respectively. 
Although we consider complex pencils, the results of this section also hold for real pencils.

4.1. Perturbation of pencils arising in continuous-time control. The first application that we discuss are matrix pencils of the form (1.4) where we perturb only the blocks $E, A, B, Q, S, R$ but such that $Q$ and $R$ stay Hermitian, i.e., we consider the case

$$
H=\left[\begin{array}{ccc}
0 & A & B \\
A^{H} & Q & S \\
B^{H} & S^{H} & R
\end{array}\right], N=\left[\begin{array}{ccc}
0 & E & 0 \\
-E^{H} & 0 & 0 \\
0 & 0 & 0
\end{array}\right]
$$

with $A, Q, E \in \mathbb{C}^{n, n}, B, S^{H} \in \mathbb{C}^{n, m}, R \in \mathbb{C}^{m, m}, Q=Q^{H}, R=R^{H}$ and we assume that $E$ is invertible. The perturbation matrices are

$$
\Delta H=\left[\begin{array}{ccc}
0 & \Delta A & \Delta B \\
(\Delta A)^{H} & \Delta Q & \Delta S \\
(\Delta B)^{H} & (\Delta S)^{H} & \Delta R
\end{array}\right], \Delta N:=\left[\begin{array}{ccc}
0 & \Delta E & 0 \\
-(\Delta E)^{H} & 0 & 0 \\
0 & 0 & 0
\end{array}\right]
$$

where the dimensions are analogous and where we assume that $(\Delta Q)^{H}=\Delta Q$, $(\Delta R)^{H}=\Delta R$ and that $E+\Delta E$ is still invertible. The pencils $(H, N)$ and $(H+$ $\Delta H, N+\Delta N)$ are then both Hermitian/skew Hermitian pencils and we are interested in the set $\mathbb{C}_{g}:=\mathbb{C} \backslash\{z \in \mathbb{C}: \operatorname{Re}(z)=0\}$. Hence, the quantity of interest is the smallest value $|t|, t \in \mathbb{R}$, such that $(H+t \Delta H, N+t \Delta N)$ has a purely imaginary eigenvalue. In view of Lemma 3.1 (with $z=i \gamma, \gamma \in \mathbb{R}$ ) this is equivalent to finding the smallest $|\gamma|$ such that the matrix $(\Delta H-i \gamma \Delta N)(H-i \gamma N)^{-1}$ has a non-zero real eigenvalue.

Evidently, we have the following expressions for Hermitian Frobenius factors $T(i \gamma)$ and $S(i \gamma)$ of the matrix $(\Delta H-i \gamma \Delta N)(H-i \gamma N)^{-1}$.

$$
\begin{aligned}
& T(i \gamma)=\left[\begin{array}{ccc}
0 & \Delta A-i \gamma \Delta E & \Delta B \\
(\Delta A-i \gamma \Delta E)^{H} & \Delta Q & \Delta S \\
(\Delta B)^{H} & (\Delta S)^{H} & \Delta R
\end{array}\right], \\
& S(i \gamma)=\left[\begin{array}{ccc}
0 & A-i \gamma E & B \\
(A-i \gamma E)^{H} & Q & S \\
B^{H} & S^{H} & R
\end{array}\right] .
\end{aligned}
$$

We may directly use Lemma 3.3 and Lemma 3.4 to obtain conditions for $(H+t \Delta H, N+$ $t \Delta N)$ to have a purely imaginary eigenvalue, as $t$ varies in $\mathbb{R}$. But the special structure of the Frobenius factors provides another condition that is more specific to the problem at hand. To obtain it, we assume without loss of generality that the matrix $[A B]$ is not a square matrix, i.e., that the matrix $B$ has at least one column.

THEOREM 4.1. Consider a matrix pencil $(H, N)$ and associated perturbations $\Delta H$ and $\Delta N$ as in (4.1) and (4.2). Let

$$
\begin{aligned}
P(t, \gamma) & :=[A-i \gamma E+t(\Delta A-i \gamma \Delta E) B+t \Delta B], \\
Z(t) & :=\left[\begin{array}{cc}
Q+t \Delta Q & S+t \Delta S \\
(S+t \Delta S)^{H} & R+t \Delta R
\end{array}\right] .
\end{aligned}
$$

Let $V(t, \gamma)$ be the set of right singular vectors of $P(t, \gamma)$ corresponding to the singular value 0 , and let $W(t, \gamma)$ be the range of $P(t, \gamma)^{H}$. 
Then for given real numbers $t \neq 0$ and $\gamma$, the purely imaginary number $i \gamma$ is an eigenvalue of the matrix pencil $(H+t \Delta H, N+t \Delta N)$ if and only if

$$
Z(t)(V(t, \gamma)) \cap W(t, \gamma) \neq \emptyset .
$$

Proof. We make use of the fact $(H+t \Delta H, N+t \Delta N), t \in \mathbb{R}$ has a purely imaginary eigenvalue $i \gamma, \gamma \in \mathbb{R}$ if and only if $-1 / t$ is an eigenvalue of the matrix $(\Delta H-i \gamma \Delta N)(H-i \gamma N)^{-1}$. Considering a Frobenius factorization

$$
(\Delta H-i \gamma \Delta N)(H-i \gamma N)^{-1}=T(i \gamma)(S(i \gamma))^{-1},
$$

it follows that $i \gamma$ is an eigenvalue of $(H+t \Delta H, N+t \Delta N)$ if and only $-1 / t$ is an eigenvalue of $T(i \gamma) S(i \gamma)^{-1}$, i.e., if and only if there exists a vector $x \neq 0$, such that $T(i \gamma) S(i \gamma)^{-1} x=-\frac{1}{t} x$. Setting $y:=S(i \gamma)^{-1} x$, this, in turn, implies that $i \gamma$, is an eigenvalue of $(H+t \Delta H, N+t \Delta N)$, if and only if there exists a vector $y \neq 0$, such that $S(i \gamma) y=-t T(i \gamma) y$. Writing down the expressions for $T(i \gamma)$, and $S(i \gamma)$, we have

$$
\left[\begin{array}{c|cc}
0 & A-i \gamma E+t(\Delta A-i \gamma \Delta E) & B+t \Delta B \\
\hline\left(A-i \gamma E+t(\Delta A-i \gamma \Delta E)^{H}\right. & Q+t \Delta Q & S+t \Delta S \\
(B+t \Delta B)^{H} & (S+t \Delta S)^{H} & R+t \Delta R
\end{array}\right] y=0 .
$$

This in turn can be written as

$$
\left[\begin{array}{cc}
0 & P(t, \gamma) \\
P(t, \gamma)^{H} & Z(t)
\end{array}\right]\left[\begin{array}{l}
y_{1} \\
y_{2}
\end{array}\right]=0
$$

Hence, we have the following system of equations.

$$
\begin{aligned}
P(t, \gamma) y_{2} & =0 \\
P(t, \gamma)^{H} y_{1}+Z(t) y_{2} & =0
\end{aligned}
$$

From the first equation we have that either $y_{2}=0$ or 0 is a singular value of $P(t, \gamma)$ and $y_{2}$ a corresponding singular vector. But as $[A B]$ is not a square matrix, neither is $P(t, \gamma)=[A-i \gamma E+t(\Delta A-i \gamma \Delta E) B+t \Delta B]$. As a consequence a nonzero vector $y_{2}$ satisfying the first equation always exists. Therefore, a necessary and sufficient condition for $i \gamma$ to be an eigenvalue of $(H+t \Delta H, N+t \Delta N)$, is that for every right singular vector $y_{2}$ of $P(t, \gamma)$ corresponding to the singular value 0 , there exists some vector $y_{1}$, such that $-P(t, \gamma)^{H} y_{1}=Z(t) y_{2}$. This implies that the matrix $Z(t)$ maps at least one right singular vector of $P(t, \gamma)$ corresponding to the singular value 0 , to the range of $P(t, \gamma)^{H}$. Since $V(t, \gamma)$ is the set of all these right singular vectors of $P(t, \gamma)$, it follows that $i \gamma$ is an eigenvalue of $(H+t \Delta H, N+t \Delta N)$, if and only if $Z(t)(V(t, \gamma)) \cap W(t, \gamma) \neq \emptyset$.

In the applications from control theory, the matrices $Q, R$ and $S$ are associated with the cost function and often these cost functions can be chosen. If this is case, then we may assume that the corresponding perturbations $\Delta Q, \Delta R$ and $\Delta S$ are all equal to zero. Under this assumption, we have the following immediate corollary of Theorem 4.1.

Corollary 4.2. Suppose that $W(t, \gamma)$ and $V(t, \gamma)$ are as in Theorem 4.1 and that $Z_{0}:=\left[\begin{array}{cc}Q & S \\ S^{H} & R\end{array}\right]$. Then the matrix pencil $(H+t \Delta H, N+t \Delta N)$ has an eigenvalue i $\gamma$ if and only if $Z_{0}(V(t, \gamma)) \cap W(t, \gamma) \neq \emptyset$. 
Proof. The proof follows immediately from Theorem 4.1 by noticing the fact that $Z(0)=Z_{0}$.

Corollary 4.2 implies that for a given fixed real number $t$, the matrices $Q, S$ and $R$ of the cost functional can be chosen in such a way that the pencil $(H+t \Delta H, N+t \Delta N)$ does not have any purely imaginary eigenvalues, cp. [15].

Corollary 4.3. If $P(t, \gamma)$ and $V(t, \gamma)$ are as in Theorem 4.1 and $Z_{0}:=$ $\left[\begin{array}{cc}Q & S \\ S^{H} & R\end{array}\right]$, then for a fixed $t \in \mathbb{R}$, the matrix pencil $(H+t \Delta H, N+t \Delta N)$ has no purely imaginary eigenvalues if and only if

$$
Z_{0}\left(\cup_{\gamma \in \mathbb{R}} V(t, \gamma)\right) \cap\left(\cup_{\gamma \in \mathbb{R}} W(t, \gamma)\right)=\emptyset .
$$

The condition of Corollary 4.3 is necessary and sufficient for a pencil $(\tilde{H}, \tilde{N}):=(H+$ $t \Delta H, N+t \Delta N)$ to have no imaginary eigenvalues. Thus, this condition generalizes well-known classical conditions that guarantee that the considered pencil has no purely imaginary eigenvalue, see e.g. $[15,17]$.

For instance, it is well known that a matrix pencil $(H, N)$, with $H$ and $N$ as in (4.1) has no purely imaginary eigenvalues if its blocks satisfy the following conditions:

(i) The matrix $R$ is positive definite and the matrix $Q-S R^{-1} S^{H}$ is positive semidefinite.

(ii) The triple $(E, A, B)$ where $A$ has size $n$, is stabilizable, i.e., for all complex numbers $\lambda$ in the closed right half plane the rank of $[A-\lambda E, B]$ is $n$.

(iii) If $Q-S R^{-1} S^{H}=C^{H} C$ is a full rank factorization of $Q-S R^{-1} S^{H}$, then $(E, A, C)$ is detectable, i.e., $\left(E^{H}, A^{H}, C^{H}\right)$ is stabilizable.

The following example shows that there exist pencils $(H, N)$ which arise from systems that are not stabilizable and detectable and yet they do not have any purely imaginary eigenvalues. This is due to the fact that they satisfy the condition given in Corollary 4.3.

EXAMPLE 4.4. Let

$$
H:=\left[\begin{array}{cc|cc|c}
0 & 0 & 2 & 3 & 2 \\
0 & 0 & 0 & 5 & 2 \\
\hline 2 & 0 & 1 & -1 & 1 \\
3 & 5 & -1 & 1 & -1 \\
\hline 2 & 2 & 1 & -1 & 5
\end{array}\right], N:=\left[\begin{array}{cc|cc|c}
0 & 0 & 1 & 0 & 0 \\
0 & 0 & 0 & 1 & 0 \\
\hline-1 & 0 & 0 & 0 & 0 \\
0 & -1 & 0 & 0 & 0 \\
\hline 0 & 0 & 0 & 0 & 0
\end{array}\right] .
$$

The eigenvalues of the pencil $(H, N)$ are $2,-2,5,-3$ and $\infty$. For this pencil we have,

$$
\begin{aligned}
& A:=\left[\begin{array}{ll}
2 & 3 \\
0 & 5
\end{array}\right], E:=\left[\begin{array}{ll}
1 & 0 \\
0 & 1
\end{array}\right], B:=\left[\begin{array}{l}
2 \\
2
\end{array}\right], \\
& Q:=\left[\begin{array}{cc}
1 & -1 \\
-1 & 1
\end{array}\right], R:=[5], S:=\left[\begin{array}{c}
1 \\
-1
\end{array}\right] .
\end{aligned}
$$

It is easy to see that $(E, A, B)$ is not stabilizable as the matrix $[A-2 I, B]=$ $\left[\begin{array}{lll}0 & 3 & 2 \\ 0 & 3 & 2\end{array}\right]$, evidently has rank 1 . We also note that

$$
\left[\begin{array}{ll}
-\frac{2}{\sqrt{5}} & \frac{2}{\sqrt{5}}
\end{array}\right]^{H}\left[\begin{array}{ll}
-\frac{2}{\sqrt{5}} & -\frac{2}{\sqrt{5}}
\end{array}\right]=\left[\begin{array}{cc}
\frac{4}{5} & -\frac{4}{5} \\
-\frac{4}{5} & \frac{4}{5}
\end{array}\right]=Q-S R^{-1} S^{H} .
$$

Setting $C:=\left[\begin{array}{ll}-\frac{2}{\sqrt{5}} & \frac{2}{\sqrt{5}}\end{array}\right]$ we observe that $(E, A, C)$ is not detectable, since $\left[A^{H}-\right.$ $\left.5 I, C^{H}\right]=\left[\begin{array}{ccc}-3 & 0 & -\frac{2}{\sqrt{5}} \\ 3 & 0 & \frac{2}{\sqrt{5}}\end{array}\right]$ has rank 1. Hence the triples $(E, A, B)$ and $(E, A, C)$ 
are also not completely controllable and completely observable, respectively, see [15]. In this case, 0 is a simple singular value of

$$
[A-i \gamma E B]:=\left[\begin{array}{ccc}
2-i \gamma & 3 & 2 \\
0 & 5-i \gamma & 2
\end{array}\right]
$$

with corresponding singular vector $v:=[-2,-2,5+i \gamma]^{T}$. The range of $[A-i \gamma E B]^{H}$ is spanned by the vectors $u_{1}:=[2+i \gamma, 3,2]^{T}$, and $u_{2}:=[0,5+i \gamma, 2]^{T}$. Therefore, $(H, N)$ has a purely imaginary eigenvalue if and only if some linear combination of $u_{1}$ and $u_{2}$ is equal to $\left[\begin{array}{cc}Q & S \\ S^{H} & R\end{array}\right] v$. This gives rise to the following equations.

$$
\begin{aligned}
(2+i \gamma) x_{1} & =5+i \gamma \\
3 x_{1}+(5+i \gamma) x_{2} & =-(5+i \gamma), \\
2 x_{1}+2 x_{2} & =5(5+i \gamma) .
\end{aligned}
$$

Eliminating $x_{1}$ and $x_{2}$ from these equations, we get the relation $\gamma=5 i \notin \mathbb{R}$.

We note that Theorem 4.1 may be generalized to the case when the zero blocks of the perturbation matrix $\Delta N$ are filled in such a way that the resulting matrix remains skew-Hermitian, that is, $\Delta N$ is replaced by $\Delta \hat{N}$ where

$$
\Delta \hat{N}:=\left[\begin{array}{lcr}
0 & \Delta E & \Delta F \\
-(\Delta E)^{H} & 0 & \Delta G \\
-(\Delta F)^{H} & -(\Delta G)^{H} & 0
\end{array}\right] .
$$

In this case, the matrix $(\Delta H-i \gamma \Delta \hat{N})(H-i \gamma N)^{-1}$ has a Frobenius factorization

$$
(\Delta H-i \gamma \Delta \hat{N})(H-i \gamma N)^{-1}=\hat{T}(i \gamma)\{S(i \gamma)\}^{-1}
$$

where

$$
\hat{T}(i \gamma)=\left[\begin{array}{lcr}
0 & \Delta A-i \gamma \Delta E & \Delta B-i \gamma \Delta F \\
(\Delta A-i \gamma \Delta E)^{H} & 0 & \Delta S-i \gamma \Delta G \\
(\Delta B-i \gamma \Delta F)^{H} & (\Delta S-i \gamma \Delta G)^{H} & 0
\end{array}\right]
$$

ThEOREM 4.5. Consider a matrix pencil $(H, N)$ and associated perturbations $\Delta H$ and $\Delta \hat{N}$ as given in (4.1), (4.2) and (4.3). Let

$$
\begin{aligned}
& \hat{P}(t, \gamma):=[A-i \gamma E+t(\Delta A-i \gamma \Delta E) B+t(\Delta B-i \gamma \Delta F)], \\
& \text { and } \hat{Z}(t, \gamma):=\left[\begin{array}{cc}
Q+t \Delta Q & S+t(\Delta S-i \gamma \Delta G) \\
(S+t(\Delta S-i \gamma \Delta G))^{H} & R+t \Delta R
\end{array}\right] \text {. }
\end{aligned}
$$

Denote by $\hat{V}(t, \gamma)$ the set of right singular vectors of $\hat{P}(t, \gamma)$ corresponding to the singular value 0 and the range of $\hat{P}(t, \gamma)^{H}$ by $\hat{W}(t, \gamma)$.

Then, for given real numbers $t \neq 0$ and $\gamma$, the purely imaginary number i $\gamma$ is an eigenvalue of the matrix pencil $(H+t \Delta H, N+t \Delta \hat{N})$ if and only if $\hat{Z}(t, \gamma)(\hat{V}(t, \gamma)) \cap$ $\hat{W}(t, \gamma) \neq \emptyset$.

Proof. The proof follows by replacing the set $T(i \gamma)$ by $\hat{T}(i \gamma)$ in the proof of Theorem 4.1.

It follows trivially, that all the results of this section also hold for those special cases when one or more of the blocks $\Delta A, \Delta B, \Delta Q, \Delta R$ and $\Delta S$ in the perturbation matrix $\Delta H$ or the block $\Delta E$ in the perturbation matrix $\Delta N$ are equal to 0 . 
4.2. The continuous-time $H_{\infty}$ problem. As mentioned in the introduction, in the case of the continuous-time, optimal $H_{\infty}$ control problem, from (1.6) we have $\Delta N=0$. Furthermore, the perturbation $\Delta H$ of $H$ has a very special structure. All its entries are zero, except for the entries of the block $\Delta R$ which is itself a special diagonal matrix, the first few entries on the main diagonal being each equal to -1 and the remaining being all equal to zero. Due to this special structure, all the eigenvalues of $(\Delta H-z \Delta N)(H-z N)^{-1}$ are real and its non-zero eigenvalues are precisely the non-zero eigenvalues of a leading principal submatrix of a Hermitian matrix whose size is the same as that of the block $R$ of $H$.

TheOrem 4.6. Let the matrices $H$ and $N$ be as in (4.1) with $R:=\left[\begin{array}{ll}R_{11} & R_{12} \\ R_{21} & R_{22}\end{array}\right]$. Let $\Delta H$ and $\Delta N$ be as in (4.2) with $\Delta A=\Delta B=\Delta Q=\Delta S=\Delta E=0$ and $\Delta R:=$ $\left[\begin{array}{cc}-I_{j} & 0 \\ 0 & 0\end{array}\right]$, where $I_{j}$ is an identity matrix of size $j$, the partition being conformal with that of $R$. Then for $\gamma \in \mathbb{R}$, all the eigenvalues of the matrix $(\Delta H-i \gamma \Delta N)(H-i \gamma N)^{-1}$ are real. In particular, the non-zero eigenvalues are the same as those of the leading principal submatrix of size $j$ of the Hermitian matrix

$$
\begin{aligned}
W(\gamma):=R^{-1} & \left(\left[\begin{array}{c}
B \\
S
\end{array}\right]^{H}\left[\begin{array}{cc}
-B R^{-1} B^{H} & A-i \gamma E-B R^{-1} S^{H} \\
\left(A-i \gamma E-B R^{-1} S^{H}\right)^{H} & Q-S R^{-1} S^{H}
\end{array}\right]^{-1} \times\right. \\
& {\left.\left[\begin{array}{c}
B \\
S
\end{array}\right]+R\right) R^{-1} . }
\end{aligned}
$$

Proof. For $\gamma \in \mathbb{R}$

$$
\begin{aligned}
& H-i \gamma N=\left[\begin{array}{ccc}
0 & A-i \gamma E & B \\
A^{H}+i \gamma E^{H} & Q & S \\
B^{H} & S^{H} & R
\end{array}\right] \\
& =\left[\begin{array}{ccc}
I & 0 & B R^{-1} \\
0 & I & S R^{-1} \\
0 & 0 & I
\end{array}\right] \times \\
& {\left[\begin{array}{ccc}
-B R^{-1} B^{H} & A-i \gamma E-B R^{-1} S^{H} & 0 \\
A^{H}+i \gamma E^{H}-S R^{-1} B^{H} & Q-S R^{-1} S^{H} & 0 \\
B^{H} & S^{H} & R
\end{array}\right] .}
\end{aligned}
$$

Therefore,

$$
\begin{gathered}
(H-i \gamma N)^{-1}=\left[\begin{array}{cc|c}
-B R^{-1} B^{H} & A-i \gamma E-B R^{-1} S^{H} & 0 \\
A^{H}+i \gamma E^{H}-S R^{-1} B^{H} & Q-S R^{-1} S^{H} & 0 \\
\hline B^{H} & S^{H} & R
\end{array}\right]^{-1} \times \\
{\left[\begin{array}{ccc}
I & 0 & -B R^{-1} \\
0 & I & -S R^{-1} \\
0 & 0 & I
\end{array}\right] .}
\end{gathered}
$$

Let $\tilde{H}:=\left[\begin{array}{cc}-B R^{-1} B^{H} & A-i \gamma E-B R^{-1} S^{H} \\ A^{H}+i \gamma E^{H}-S R^{-1} B^{H} & Q-S R^{-1} S^{H}\end{array}\right], M:=\left[\begin{array}{c}-B R^{-1} \\ -S R^{-1}\end{array}\right]$, and $Z:=\left[\begin{array}{cc}B^{H} & S^{H}\end{array}\right]$. Then

$$
(H-i \gamma N)^{-1}=\left[\begin{array}{cc}
\tilde{H} & 0 \\
Z & R
\end{array}\right]^{-1}\left[\begin{array}{cc}
I & M \\
0 & I
\end{array}\right]=\left[\begin{array}{cc}
\tilde{H}^{-1} & 0 \\
-R^{-1} Z \tilde{H}^{-1} & R^{-1}
\end{array}\right]\left[\begin{array}{cc}
I & M \\
0 & I
\end{array}\right]
$$




$$
\begin{aligned}
& =\left[\begin{array}{cc}
\tilde{H}^{-1} & \tilde{H}^{-1} M \\
-R^{-1} Z \tilde{H}^{-1} & -R^{-1} Z \tilde{H}^{-1} M+R^{-1}
\end{array}\right] \\
& =\left[\begin{array}{cc}
\tilde{H}^{-1} & \tilde{H}^{-1} M \\
M^{H} \tilde{H}^{-1} & -M^{H} \tilde{H}^{-1} M+R^{-1}
\end{array}\right],
\end{aligned}
$$

which is Hermitian, since $\tilde{H}$ is Hermitian. Then, we obtain

$$
\begin{aligned}
(\Delta H-i \gamma \Delta N)(H-i \gamma N)^{-1} & =\left[\begin{array}{cc}
0 & 0 \\
0 & \Delta R
\end{array}\right]\left[\begin{array}{cc}
\tilde{H}^{-1} & \tilde{H}^{-1} M \\
M^{H}(\tilde{H})^{-1} & M^{H} \tilde{H}^{-1} M+R^{-1}
\end{array}\right] \\
& =\left[\begin{array}{cc}
0 & 0 \\
(\Delta R) M^{H} \tilde{H}^{-1} & (\Delta R)\left(M^{H} \tilde{H}^{-1} M+R^{-1}\right)
\end{array}\right] .
\end{aligned}
$$

Therefore, the matrix $(\Delta H-i \gamma \Delta N)(H-i \gamma N)^{-1}$ has a non-zero real eigenvalue if and only if the matrix $(\Delta R)\left(M^{H} \tilde{H}^{-1} M+R^{-1}\right)$ has a non-zero real eigenvalue. Replacing $M$ by $\left[\begin{array}{l}-B R^{-1} \\ -S R^{-1}\end{array}\right]$ we have

$$
\begin{aligned}
(\Delta R)\left(M^{H} \tilde{H}^{-1} M+R^{-1}\right) & =(\Delta R) R^{-1}\left(\left[\begin{array}{c}
B \\
S
\end{array}\right]^{H} \tilde{H}^{-1}\left[\begin{array}{c}
B \\
S
\end{array}\right]+R\right) R^{-1} \\
& =(\Delta R) W(\gamma) .
\end{aligned}
$$

Note that since the matrices $R$ and $Q$ are Hermitian, $W(\gamma)$ is also Hermitian and hence all its eigenvalues are real. Let

$$
W(\gamma):=\left[\begin{array}{ll}
W_{11}(\gamma) & W_{12}(\gamma) \\
W_{21}(\gamma) & W_{22}(\gamma)
\end{array}\right]
$$

be a partition of $W(\gamma)$, conformal with that of $\Delta R$. In view of the structure of $\Delta R$, it follows that

$$
(\Delta R) W(\gamma)=\left[\begin{array}{cc}
-W_{11}(\gamma) & -W_{12}(\gamma) \\
0 & 0
\end{array}\right]
$$

Hence $(\Delta H-i \gamma \Delta N)(H-i \gamma N)^{-1}$ has a non-zero real eigenvalue if and only if the block $W_{11}(\gamma)$ has a non-zero real eigenvalue. The proof follows from the fact that $W_{11}(\gamma)$ is Hermitian.

From Theorem 4.6 it follows that for the continuous time $H_{\infty}$ control problem we have $\operatorname{sep}_{\mathbb{R}}(z, \Delta H, \Delta N)=\left\{r\left((\Delta H-z \Delta N)(H-z N)^{-1}\right)\right\}^{-1}$ for all purely imaginary complex numbers $z$. However, for these problems we are interested only in positive values of the parameter $t$ for which $(H+t \Delta H, N+t \Delta N)$ has a purely imaginary eigenvalue. The following immediate corollary of Theorem 4.6 suggests a procedure for obtaining the exact value of the smallest positive parameter $t$ for which the pencil $(H+t \Delta H, N+t \Delta N)$ has a purely imaginary eigenvalue or an upper or lower bound of this value.

Corollary 4.7. Let $H, N, \Delta H$ and $\Delta N$ be as in Theorem 4.6. The smallest positive parameter $t$ for which $(H+t \Delta H, N+t \Delta N)$ has a purely imaginary eigenvalue is, say $t=t_{0}$, if and only if

(i) $1 / t_{0}$ is the smallest value of $\epsilon$ for which the set

$$
L(\epsilon, \Delta H, \Delta N):=\left\{z \in \mathbb{C} \backslash \sigma(H, N): r\left((\Delta H-z \Delta N)(H-z N)^{-1}\right)=\epsilon^{-1}\right\}
$$


touches the imaginary axis and

(ii) $1 / t_{0}$ is an eigenvalue of largest magnitude of the matrix $W_{11}\left(\gamma_{0}\right)$, given by (4.4), $i \gamma_{0}$ being the point at which $L\left(1 / t_{0}, \Delta H, \Delta N\right)$ touches the imaginary axis.

If all the eigenvalues of $W_{11}\left(\gamma_{0}\right)$ are negative real numbers, then the smallest positive parameter $t$ for which $(H+t \Delta H, N+t \Delta N)$ has a purely imaginary eigenvalue is larger than $\left\{r\left(W_{11}\left(\gamma_{0}\right)\right)\right\}^{-1}$.

If $W_{11}\left(\gamma_{0}\right)$ has positive eigenvalues but none of them is equal to $r\left(W_{11}\left(\gamma_{0}\right)\right)$, and $\alpha$ is the largest among these eigenvalues, then the smallest positive parameter $t$ for which $(H+t \Delta H, N+t \Delta N)$ has a purely imaginary eigenvalue is less than or equal to $1 / \alpha$.

Proof. The proof follows immediately from Theorem 4.6 in view of the fact that given a positive real number $t_{0}, i \gamma_{0}$ is a purely imaginary eigenvalue of $\left(H+t_{0} \Delta H, N+\right.$ $\left.t_{0} \Delta N\right)$ if and only if $1 / t_{0}$ is an eigenvalue of $W_{11}\left(\gamma_{0}\right)$.

In this section we have discussed linear perturbation theory for structured pencils arising in continuous-time control theory. In the next section we discuss analogous results for discrete-time control problems.

5. Perturbation of structured pencils arising from discrete-time control. There is a well-known analogy between continuous and discrete-time linear quadratic optimal control problems, given by the Cayley transformation, see [17, 18]. Thus, we expect similar results for the discrete time case. For these problems the pencils have the following structures.

$$
\begin{gathered}
H:=\left[\begin{array}{ccc}
0 & A & B \\
-E^{H} & Q & S \\
0 & S^{H} & R
\end{array}\right], N:=\left[\begin{array}{ccc}
0 & E & 0 \\
-A^{H} & 0 & 0 \\
-B^{H} & 0 & 0
\end{array}\right], \\
\Delta H:=\left[\begin{array}{ccc}
0 & \Delta A & \Delta B \\
-(\Delta E)^{H} & \Delta Q & \Delta S \\
0 & (\Delta S)^{H} & \Delta R
\end{array}\right], \Delta N:=\left[\begin{array}{ccc}
0 & \Delta E & 0 \\
-(\Delta A)^{H} & 0 & 0 \\
-(\Delta B)^{H} & 0 & 0
\end{array}\right]
\end{gathered}
$$

where again $Q^{H}=Q, R^{H}=R,(\Delta Q)^{H}=\Delta Q$, and $(\Delta R)^{H}=\Delta R$ have the same dimensions as in (4.1). Although we consider complex matrices, the results of this section are true for real matrices as well.

In this case $\mathbb{C}_{g}:=\{z \in \mathbb{C}:|z| \neq 1\}$, and the smallest $|t|, t \in \mathbb{R}$ such that the perturbed pencil $(H+t \Delta H, N+t \Delta N)$ has an eigenvalue $z \in \mathbb{C},|z|=1$, is the quantity of interest for these problems. This is equivalent to the matrix $(\Delta H-z \Delta N)(H-$ $z N)^{-1}$ having a real eigenvalue for some $z \in \mathbb{C}$ such that $|z|=1$. We show first that for any $z \in \mathbb{C}$ on the unit circle, Hermitian Frobenius factors $T(z)$ and $S(z)$ of the matrix $(\Delta H-z \Delta N)(H-z N)^{-1}$ may be obtained from the matrices $\Delta H-z \Delta N$ and $H-z N$ by a simple scaling.

TheOREM 5.1. Let the matrices $H, N, \Delta H$ and $\Delta N$ be as given in (5.1) and (5.2). Then for $z \in \mathbb{C},|z|=1$,

$$
\begin{aligned}
& (\Delta H-z \Delta N)(H-z N)^{-1} \\
= & {\left[\begin{array}{ccc}
0 & \Delta A-z \Delta E & \Delta B \\
(\Delta A-z \Delta E)^{H} & \Delta Q & \Delta S \\
(\Delta B)^{H} & (\Delta S)^{H} & \Delta R
\end{array}\right]\left[\begin{array}{ccc}
0 & A-z E & B \\
(A-z E)^{H} & Q & S \\
B^{H} & S^{H} & R
\end{array}\right]^{-1} . }
\end{aligned}
$$


Proof. For $|z|=1$, we have,

$$
\begin{aligned}
H-z N & =\left[\begin{array}{ccc}
0 & A-z E & B \\
z(A-z E)^{H} & Q & S \\
z B^{H} & S^{H} & R
\end{array}\right] \\
& =\left[\begin{array}{ccc}
0 & A-z E & B \\
(A-z E)^{H} & Q & S \\
B^{H} & S^{H} & R
\end{array}\right]\left[\begin{array}{ccc}
z I & & 0 \\
0 & I & 0 \\
0 & 0 & I
\end{array}\right] .
\end{aligned}
$$

Similarly,

$$
\Delta H-z \Delta N=\left[\begin{array}{ccc}
0 & \Delta A-z \Delta E & \Delta B \\
(\Delta A-z \Delta E)^{H} & \Delta Q & \Delta S \\
(\Delta B)^{H} & (\Delta S)^{H} & \Delta R
\end{array}\right]\left[\begin{array}{ccc}
z I & 0 & 0 \\
0 & I & 0 \\
0 & 0 & I
\end{array}\right] .
$$

The proof follows from the fact that the matrix $\left[\begin{array}{ccc}z I & 0 & 0 \\ 0 & I & 0 \\ 0 & 0 & I\end{array}\right]$ is unitary, since $|z|=1$. $\square$

Hence, for all $z \in \mathbb{C}$ such that $|z|=1$, we get Hermitian Frobenius factors $T(z)$ and $S(z)$ of $(\Delta H-z \Delta N)(H-z N)^{-1}$ as

$$
\begin{aligned}
T(z) & :=\left[\begin{array}{ccc}
0 & \Delta A-z \Delta E & \Delta B \\
(\Delta A-z \Delta E)^{H} & \Delta Q & \Delta S \\
(\Delta B)^{H} & (\Delta S)^{H} & \Delta R
\end{array}\right], \\
S(z) & :=\left[\begin{array}{ccc}
0 & A-z E & B \\
(A-z E)^{H} & Q & S \\
B^{H} & S^{H} & R
\end{array}\right] .
\end{aligned}
$$

Thus, given a complex number $z$ lying on the unit circle, the results of Section 3 may be applied to these Frobenius factors to obtain necessary and sufficient conditions for the matrix $(\Delta H-z \Delta N)(H-z N)^{-1}$ to have a real eigenvalue. This in turn gives us necessary and sufficient conditions for the matrix pencil $(H+t \Delta H, N+t \Delta N)$ to have an eigenvalue on the unit circle. However, as in the continuous time case, the special structure of the Frobenius factors lead to another necessary and sufficient condition for the pencil $(H+t \Delta H, N+t \Delta N)$ to have an eigenvalue on the unit circle on the lines of Theorem 4.1.

THEOREM 5.2. Consider a matrix pencil $(H, N)$ and associated perturbations $\Delta H$ and $\Delta N$ as in (5.1) and (5.2). Let $z \in \mathbb{C}$ such that $|z|=1$, and

$$
\begin{aligned}
P(t, z) & :=[A-z E+t(\Delta A-z \Delta E) B+t \Delta B], \\
Z(t) & :=\left[\begin{array}{cc}
Q+t \Delta Q & S+t \Delta S \\
(S+t \Delta S)^{H} & R+t \Delta R
\end{array}\right] .
\end{aligned}
$$

Let $V(t, z)$ be the set of right singular vectors of $P(t, z)$ corresponding to the singular value 0 and let $W(t, z)$ be the range of $P(t, z)^{H}$.

Then, for given a real number $t \neq 0$ and a complex number $z \in \mathbb{C},|z|=1$ is an eigenvalue of the matrix pencil $(H+t \Delta H, N+t \Delta N)$ if and only if $Z(t)(V(t, z)) \cap$ $W(t, z) \neq \emptyset$.

Proof. The proof follows by replacing $i \gamma$ by $z \in \mathbb{C},|z|=1$, in the proof of Theorem 4.1. 
As in the continuous time case, if we assume that the matrices $Q, S$, and $R$ which are associated with the cost function are unperturbed, that is, if $\Delta Q=\Delta S=\Delta R=0$, then we have the following corollary to Theorem 5.2. It characterizes the choice of a cost function such that given $t \in \mathbb{R}$, and $z \in \mathbb{C}$ such that $|z|=1, z$ is not an eigenvalue of $(H+t \Delta H, N+t \Delta N)$.

Corollary 5.3. Let $W(t, z)$, and $V(t, z)$ be as in Theorem 5.2 and let $Z_{0}:=$ $\left[\begin{array}{cc}Q & S \\ S^{H} & R\end{array}\right]$. The matrix pencil $(H+t \Delta H, N+t \Delta N)$ has an eigenvalue $z$ with $|z|=1$ if and only if $Z_{0}(V(t, z)) \cap W(t, z) \neq \emptyset$. $Z_{0} . \quad$ r

Proof. The proof follows immediately from Theorem 5.2 by the fact that $Z(0)=$

The next corollary provides a characterization of all cost functions such that for a fixed $t \in \mathbb{R}$, the pencil $(H+t \Delta H, N+t \Delta N)$ does not have any eigenvalues on the unit circle.

Corollary 5.4. Suppose that $P(t, z)$ and $V(t, z)$ are as in Theorem 5.2 and that $Z_{0}:=\left[\begin{array}{cc}Q & S \\ S^{H} & R\end{array}\right]$. Then, for a fixed $t \in \mathbb{R}$, the matrix pencil $(H+t \Delta H, N+t \Delta N)$ has no eigenvalues on the unit circle if and only if

$$
Z_{0}\left(\cup_{|z|=1} V(t, z)\right) \cap\left(\cup_{|z|=1} W(t, z)\right)=\emptyset
$$

The results of this section also hold if the perturbation matrices $\Delta H$ and $\Delta N$ are replaced by the matrices $\Delta \hat{H}$ and $\Delta \hat{N}$, respectively, which are given by

$$
\Delta \hat{H}:=\left[\begin{array}{lcc}
0 & \Delta A & \Delta B \\
-(\Delta E)^{H} & \Delta Q & \Delta S \\
-(\Delta F)^{H} & (\Delta S)^{H} & \Delta R
\end{array}\right], \Delta \hat{N}:=\left[\begin{array}{lcr}
0 & \Delta E & \Delta F \\
-(\Delta A)^{H} & 0 & 0 \\
-(\Delta B)^{H} & 0 & 0
\end{array}\right]
$$

Then for $z \in \mathbb{C}$ such that $|z|=1,(\Delta \hat{H}-z \Delta \hat{N})(H-z N)^{-1}=\hat{T}(z) S(z)^{-1}$, is a Frobenius factorization of $(\Delta \hat{H}-z \Delta \hat{N})(H-z N)^{-1}$, where

$$
\hat{T}(z):=\left[\begin{array}{ccc}
0 & \Delta A-z \Delta E & \Delta B-z \Delta F \\
(\Delta A-z \Delta E)^{H} & \Delta Q & \Delta S \\
(\Delta B-z \Delta F)^{H} & (\Delta S)^{H} & \Delta R
\end{array}\right] .
$$

With these new perturbation matrices, Theorem 5.2 takes the following form.

THEOREM 5.5. Consider a matrix pencil $(H, N)$ and associated perturbations $\Delta \hat{H}$ and $\Delta \hat{N}$ as given in (5.1) and (5.3). Let

$$
\begin{aligned}
\hat{P}(t, z) & :=[A-z E+t(\Delta A-z \Delta E) \\
Z(t) & :=\left[\begin{array}{cc}
Q+t \Delta Q & S+t(\Delta B-z \Delta F)], \\
(S+t(\Delta S))^{H} & R+t \Delta R
\end{array}\right] .
\end{aligned}
$$

Let $\hat{V}(t, z)$ be the set of right singular vectors of $\hat{P}(t, z)$ corresponding to the singular value 0 , and let $\hat{W}(t, z)$ be the range of $\hat{P}(t, z)^{H}$.

Then, for a given real number $t \neq 0$, a complex number $z \in \mathbb{C}$ with $|z|=1$ is an eigenvalue of the matrix pencil $(H+t \Delta \hat{H}, N+t \Delta \hat{N})$ if and only if $Z(t)(\hat{V}(t, z)) \cap$ $\hat{W}(t, z) \neq \emptyset$.

Proof. The proof follows by using arguments similar to those of Theorem 4.1 with the matrix $P(t, i \gamma)$ being replaced by $\hat{P}(t, z)$. 
Given $t \in \mathbb{R}$ and $z \in \mathbb{C},|z|=1$, the following corollary provides a characterization of the cost function such that $z \notin \sigma(H+t \Delta \hat{H}, N+t \Delta \hat{N})$.

Corollary 5.6. Let $\hat{P}(t, z), \hat{V}(t, z)$ and $\hat{W}(t, z)$ be as in Theorem 5.5 and let $Z_{0}:=\left[\begin{array}{cc}Q & S \\ S^{H} & R\end{array}\right]$. Then, for a fixed real number $t$ and $z \in \mathbb{C}$, such that $|z|=1$, we have $z \notin \sigma(H+t \Delta \hat{H}, N+t \Delta \hat{N})$ if and only if $Z_{0}(\hat{V}(t, z)) \cap \hat{W}(t, z) \neq \emptyset$.

Finally we have a characterization of the cost function such that for a given $t \in \mathbb{R}$, the pencil $(H+t \Delta \hat{H}, N+t \Delta \hat{N})$ has no eigenvalues on the unit circle.

Corollary 5.7. Let $\hat{P}(t, z), \hat{V}(t, z)$ and $\hat{W}(t, z)$ be as in Theorem 5.5 and let $Z_{0}:=\left[\begin{array}{cc}Q & S \\ S^{H} & R\end{array}\right]$. Then for a fixed real number $t$, the pencil $(H+t \Delta \hat{H}, N+t \Delta \hat{N})$ has no eigenvalues on the unit circle if and only if

$$
Z_{0}\left(\cup_{|z|=1} \hat{V}(t, z)\right) \cap\left(\cup_{|z|=1} \hat{W}(t, z)\right) \neq \emptyset .
$$

Note that all results hold if any one or more of the blocks in the perturbation matrices $\Delta H$ and $\Delta N$ are equal to 0 .

6. The discrete-time $H_{\infty}$ problem. In the discrete-time optimal $H_{\infty}$ control problem, the matrices $H$ and $N$ of the pencil $(H, N)$ are also given by (5.1). But as in the case of its continuous time analogue, the perturbations $\Delta N$ and $\Delta H$ are very special. From equation (1.7) we have $\Delta N=0$ and all the blocks of $\Delta H$ are zero except for $\Delta R$ which is a special diagonal matrix. Only the first few diagonal entries of $\Delta R$ are non-zero and these are each equal to -1 . We show that given $|z|=1$, the matrix all the eigenvalues of $(\Delta H-z \Delta N)(H-z N)^{-1}$ are real and the non-zero eigenvalues are precisely the same as those of a leading principal submatrix of a Hermitian matrix which is of the same size as the block $R$ of $H$.

Theorem 6.1. Let the matrices $H$ and $N$ be as in (5.1) with $R:=\left[\begin{array}{ll}R_{11} & R_{12} \\ R_{21} & R_{22}\end{array}\right]$. Let $\Delta H$ and $\Delta N$ be as in (5.2) with $\Delta A=\Delta B=\Delta Q=\Delta S=\Delta E=0$ and $\Delta R:=\left[\begin{array}{cc}-I_{j} & 0 \\ 0 & 0\end{array}\right]$, where $I_{j}$ is an identity matrix of size $j$, the partition being conformal with that of $R$. Then, for $z \in \mathbb{C}$ such that $|z|=1$, all eigenvalues of the matrix $(\Delta H-z \Delta N)(H-z N)^{-1}$ are real. In particular its non-zero real eigenvalues are the same as those of the leading principal submatrix of size $j$ of the Hermitian matrix

$$
\begin{aligned}
W(z):=R^{-1} & \left(\left[\begin{array}{c}
B \\
S
\end{array}\right]^{H}\left[\begin{array}{cc}
-B R^{-1} B^{H} & A-z E-B R^{-1} S^{H} \\
\left(A-z E-B R^{-1} S^{H}\right)^{H} & Q-S R^{-1} S^{H}
\end{array}\right]^{-1} \times\right. \\
& {\left.\left[\begin{array}{c}
B \\
S
\end{array}\right]+R\right) R^{-1} . }
\end{aligned}
$$

Proof. Since $R$ and $Q$ are Hermitian, it is clear that $W(z)$ is a Hermitian matrix. For $z \in \mathbb{C},|z|=1$, we have,

$$
\begin{aligned}
H-z N & =\left[\begin{array}{ccc}
0 & A-z E & B \\
-E^{H}+z A^{H} & Q & S \\
z B^{H} & S^{H} & R
\end{array}\right] \\
& =\left[\begin{array}{ccc}
I & 0 & B R^{-1} \\
0 & I & S R^{-1} \\
0 & 0 & I
\end{array}\right] \times\left[\begin{array}{ccc}
-z B R^{-1} B^{H} & A-z E-B R^{-1} S^{H} & 0 \\
z\left(A-z E-B R^{-1} S^{H}\right)^{H} & Q-S R^{-1} S^{H} & 0 \\
z B^{H} & S^{H} & R
\end{array}\right] .
\end{aligned}
$$


Therefore,

$$
\begin{aligned}
& (H-z N)^{-1}=\left[\begin{array}{ccc}
-z B R^{-1} B^{H} & A-z E-B R^{-1} S^{H} & 0 \\
-E^{H}+z A^{H}-z S R^{-1} B^{H} & Q-S R^{-1} S^{H} & 0 \\
z B^{H} & S^{H} & R
\end{array}\right]^{-1} \times \\
& {\left[\begin{array}{ccc}
I & 0 & -B R^{-1} \\
0 & I & -S R^{-1} \\
0 & 0 & I
\end{array}\right]}
\end{aligned}
$$

Let

$$
\tilde{H}=\left[\begin{array}{cc}
-z B R^{-1} B^{H} & A-z E-B R^{-1} S^{H} \\
z\left(A^{H}-\bar{z} E^{H}-S R^{-1} B^{H}\right) & Q-S R^{-1} S^{H}
\end{array}\right], M=\left[\begin{array}{c}
-B R^{-1} \\
-S R^{-1}
\end{array}\right]
$$

and $Z=\left[\begin{array}{ll}z B^{H} & S^{H}\end{array}\right]$. Then,

$$
\begin{aligned}
(H-z N)^{-1} & =\left[\begin{array}{cc}
\tilde{H} & 0 \\
Z & R
\end{array}\right]^{-1}\left[\begin{array}{cc}
I & M \\
0 & I
\end{array}\right]=\left[\begin{array}{cc}
\tilde{H}^{-1} & 0 \\
-R^{-1} Z \tilde{H}^{-1} & R^{-1}
\end{array}\right]\left[\begin{array}{cc}
I & M \\
0 & I
\end{array}\right] \\
& =\left[\begin{array}{cc}
\tilde{H}^{-1} & \tilde{H}^{-1} M \\
-R^{-1} Z \tilde{H}^{-1} & -R^{-1} Z \tilde{H}^{-1} M+R^{-1}
\end{array}\right] .
\end{aligned}
$$

Let $\tilde{J}=\left[\begin{array}{cc}\bar{z} I & 0 \\ 0 & I\end{array}\right]$ where the partitioning is conformal with that of $M$. Since $|z|=$ 1 , it is clear that $\tilde{J}$ is unitary and $R^{-1} Z=-M^{H} \tilde{J}^{-1}$. This gives $R^{-1} Z \tilde{H}^{-1}=$ $-M^{H}(\tilde{H} \tilde{J})^{-1}$ and hence,

$$
(H-z N)^{-1}=\left[\begin{array}{cc}
\tilde{H}^{-1} & \tilde{H}^{-1} M \\
M^{H}(\tilde{H} \tilde{J})^{-1} & M^{H}(\tilde{H} \tilde{J})^{-1} M+R^{-1}
\end{array}\right] .
$$

Therefore,

$$
(\Delta H-z \Delta N)(H-z N)^{-1}=\left[\begin{array}{cc}
0 & 0 \\
0 & \Delta R
\end{array}\right]\left[\begin{array}{cc}
\tilde{H}^{-1} & \tilde{H}^{-1} M \\
M^{H}(\tilde{H} \tilde{J})^{-1} & M^{H}(\tilde{H} \tilde{J})^{-1} M+R^{-1}
\end{array}\right]
$$

This shows that the matrix $(\Delta H-z \Delta N)(H-z N)^{-1}$ has non-zero real eigenvalues if and only if the matrix

$$
(\Delta R)\left(M^{H}(\tilde{H} \tilde{J})^{-1} M+R^{-1}\right)
$$

has non-zero real eigenvalues or equivalently

$$
(\Delta R) R^{-1}\left(\left[\begin{array}{c}
B \\
S
\end{array}\right]^{H}(\tilde{H} \tilde{J})^{-1}\left[\begin{array}{c}
B \\
S
\end{array}\right]+R\right) R^{-1}
$$

has non-zero real eigenvalues. Since,

$$
\tilde{H} \tilde{J}=\left[\begin{array}{cc}
-B R^{-1} B^{H} & A-z E-B R^{-1} S^{H} \\
\left(A-z E-B R^{-1} S^{H}\right)^{H} & Q-S R^{-1} S^{H}
\end{array}\right],
$$

we have,

$$
(\Delta R) R^{-1}\left(\left[\begin{array}{c}
B \\
S
\end{array}\right]^{H}(\tilde{H} \tilde{J})^{-1}\left[\begin{array}{c}
B \\
S
\end{array}\right]+R\right) R^{-1}=(\Delta R) W(z)
$$


Let

$$
W(z):=\left[\begin{array}{ll}
W_{11}(z) & W_{12}(z) \\
W_{21}(z) & W_{22}(z)
\end{array}\right]
$$

be a partition of $W(z)$ conformal with that of $\Delta R$. In view of the structure of $\Delta R$, we have,

$$
(\Delta R) W(z)=\left[\begin{array}{cc}
-W_{11}(z) & W_{12}(z) \\
0 & 0
\end{array}\right] .
$$

Hence the non-zero real eigenvalues of $(\Delta H-z \Delta N)(H-z N)^{-1}$ are the same as those of $-W_{11}(z)$ and the proof follows from the fact that $W_{11}(z)$ is Hermitian.

By Theorem 6.1, it is clear that every point on the unit circle becomes an eigenvalue of $(H+t \Delta H, N+t \Delta N)$ for some real number $t$. However, as in the case of the continuous time $H_{\infty}$ problem, we are interested only in the positive values of the parameter $t$ for which the pencil $(H+t \Delta H, N+t \Delta N)$ has eigenvalues on the unit circle. Since, for any $z \notin \sigma(H, N)$, we have, $z \in \sigma(H+t \Delta H, N+t \Delta N)$ if and only if $-1 / t \in \sigma(\Delta H-z \Delta N)(H-z N)^{-1}$, it follows from Theorem 6.1 that there exists $t>0$ such that some $z \in \mathbb{C}$, with $|z|=1$ is an eigenvalue of $(H+t \Delta H, N+t \Delta N)$ if and only if the matrix $W_{11}(z)$ has a positive eigenvalue. This suggests the following procedure for finding the smallest positive number $t$ for which $(H+t \Delta H, N+t \Delta N)$ has an eigenvalue on the unit circle on the lines of Corollary 4.7.

Corollary 6.2. Let $H, N, \Delta H$ and $\Delta N$ be as in Theorem 6.1. The smallest positive parameter $t$ for which $(H+t \Delta H, N+t \Delta N)$ has an eigenvalue $z$ with $|z|=1$ is say $t=t_{0}$, if and only if

(i) $1 / t_{0}$ is the smallest value of $\epsilon$ for which the set

$$
L(\epsilon, \Delta H, \Delta N):=\left\{z \in \mathbb{C} \backslash \sigma(H, N): r\left((\Delta H-z \Delta N)(H-z N)^{-1}\right)=\epsilon^{-1}\right\}
$$

touches the imaginary axis and

(ii) $1 / t_{0}$ is an eigenvalue with largest magnitude of the matrix $W_{11}\left(z_{0}\right)$, given by (6.1), $z_{0}$ being the point at which $L\left(1 / t_{0}, \Delta H, \Delta N\right)$ touches the unit circle.

If all the eigenvalues of $W_{11}\left(z_{0}\right)$ are negative real numbers, then the smallest positive parameter $t$, for which $(H+t \Delta H, N+t \Delta N)$ has an eigenvalue on the unit circle, is larger than $\left\{r\left(W_{11}\left(z_{0}\right)\right)\right\}^{-1}$.

If $W_{11}\left(z_{0}\right)$ has positive eigenvalues but none of them is equal to $r\left(W_{11}\left(z_{0}\right)\right)$, and $\alpha$ is the largest among these eigenvalues, then the smallest positive parameter $t$ for which $(H+t \Delta H, N+t \Delta N)$ has an eigenvalue on the unit circle is less than or equal to $1 / \alpha$.

Proof. The proof is an immediate consequence of Theorem 6.1.

7. Conclusion and future work. We have studied the effect of linear perturbations on several structured matrix pencils arising in control theory. These include skew-symmetric/symmetric pencils arising in the computation of optimal $H_{\infty}$ control and linear quadratic control for continuous and discrete time systems. We have given characterizations when these pencils have eigenvalues on the imaginary axis or the unit circle, respectively.

But several important questions remain open. Among these are a characterization of the Kronecker structure associated with eigenvalues on the imaginary axis and to develop numerical methods for the efficient computation of the smallest perturbations that move eigenvalues to the imaginary axis or unit circle, respectively. We will address these issues in our future work. 


\section{REFERENCES}

[1] R. Alam and S. Bora. Stability of eigenvalues and spectral decompositions under linear perturbation. Linear Algebra Appl., 364:189-211, 2003.

[2] E. Anderson, Z. Bai, C.H. Bischof, J.M. Demmel, J.J. Dongarra, J.J. Du Croz, A. Greenbaum, S.J. Hammarling, A. McKenney, S. Ostrouchov, and D.C. Sorensen. LAPACK Users, Guide. SIAM, Philadelphia, PA, second edition, 1994.

[3] H. Baumgärtel. Analytic Perturbation Theory for Matrices and Operators. Birkhäuser, Philadelphia, PA, 1985.

[4] P. Benner, R. Byers, V. Mehrmann, and H. Xu. Numerical methods for linear quadratic and $H_{\infty}$ control problems. In G. Picci and D.S. Gillian, editors, Dynamical Systems, Control, Coding, Computer Vision. Progress in Systems and Control Theory, Vol. 25, pages 203222, Basel, 1999. Birkhäuser Verlag.

[5] P. Benner, R. Byers, V. Mehrmann, and H. Xu. Robust method for robust control. Preprint 2004-6, Institut für Mathematik, TU Berlin, D-10623 Berlin, FRG, 2004.

[6] A. Bunse-Gerstner, R. Byers, V. Mehrmann, and N.K. Nichols. Feedback design for regularizing descriptor systems. Linear Algebra Appl., 299:119-151, 1999.

[7] R. Byers, T. Geerts, and V. Mehrmann. Descriptor systems without controllability at infinity. SIAM J. Cont. Optim., 35:462-479, 1997.

[8] G. Freiling, V. Mehrmann, and H. Xu. Existence, uniqueness and parametrization of Lagrangian invariant subspaces. SIAM J. Matrix Anal. Appl., 23:1045-1069, 2002.

[9] G.H. Golub and C.F. Van Loan. Matrix Computations. The Johns Hopkins University Press, Baltimore, third edition, 1996.

[10] M. Green and D.J.N Limebeer. Linear Robust Control. Prentice-Hall, Englewood Cliffs, NJ, 1995.

[11] D. Hinrichsen and A.J. Pritchard. Mathematical Systems Theory I. Modelling, State Space Analysis, Stability and Robustness. Springer Verlag, Berlin, 2005.

[12] T. Kailath. Linear Systems. Prentice-Hall, Englewood Cliffs, NJ, 1980.

[13] T. Kato. Perturbation Theory for Linear Operators. Springer-Verlag, New York, NY, 1966.

[14] M.M. Konstantinov, V. Mehrmann, and P.Hr. Petkov. Perturbation analysis for the Hamiltonian Schur form. SIAM J. Matrix Anal. Appl., 23:387-424, 2002.

[15] P. Lancaster and L. Rodman. The Algebraic Riccati Equation. Oxford University Press, Oxford, 1995.

[16] P. Lancaster and M. Tismenetsky. The Theory of Matrices. Academic Press, Orlando, FL, 2nd edition, 1985

[17] V. Mehrmann. The Autonomous Linear Quadratic Control Problem, Theory and Numerical Solution. Number 163 in Lecture Notes in Control and Information Sciences. SpringerVerlag, Heidelberg, July 1991.

[18] V. Mehrmann. A step towards a unified treatment of continuous and discrete time control problems. Linear Algebra Appl., 241-243:749-779, 1996.

[19] M.L. Overton and P. Van Dooren. On computing the complex passivity radius. Technical Report Submitted for publication, CESAME, Univ. Cath. de Louvain, Belgium, 2005.

[20] L.S. Pontryagin, V. Boltyanskii, R. Gamkrelidze, and E. Mishenko. The Mathematical Theory of Optimal Processes. Interscience, New York, 1962.

[21] A.C.M. Ran and L. Rodman. Stability of invariant maximal semidefinite subspaces. Linear Algebra Appl., 62:51-86, 1984.

[22] A.C.M. Ran and L. Rodman. Stability of invariant Lagrangian subspaces i. Operator Theory: Advances and Applications (I. Gohberg ed.), 32:181-218, 1988.

[23] A.C.M. Ran and L. Rodman. Stability of invariant Lagrangian subspaces ii. Operator Theory: Advances and Applications ( H.Dym, S. Goldberg, M.A. Kaashoek and P. Lancaster eds.), 40:391-425, 1989.

[24] G.W. Stewart and J.-G. Sun. Matrix Perturbation Theory. Academic Press, New York, 1990.

[25] F. Uhlig. Inertia and eigenvalue relations between symmetrized and symmetrizing matrices for the real and the general field case. Linear Algebra Appl., 35:203-226, 1981.

[26] P. Van Dooren. The generalized eigenstructure problem in linear system theory. IEEE Trans. Automat. Control, AC-26:111-129, 1981.

[27] P. Van Dooren. A generalized eigenvalue approach for solving Riccati equations. SIAM J. Sci. Statist. Comput., 2:121-135, 1981.

[28] K. Zhou, J.C. Doyle, and K. Glover. Robust and Optimal Control. Prentice-Hall, Upper Saddle River, NJ, 1995. 